\title{
MOTIVAÇÃO ACADÊMICA E PERCEPÇÃO DE COMPETÊNCIA PROFISSIONAL DE ACADÊMICOS DE EDUCAÇÃO FÍSICA
}

\author{
José Roberto Andrade do Nascimento Júnior \\ Universidade Federal do Vale do São Francisco, Petrolina, Pernambuco, Brasil. \\ Carla Thamires Laranjeira Granja \\ Universidade Federal do Vale do São Francisco, Petrolina, Pernambuco, Brasil. \\ Eliakim Cerqueira da Silva \\ Universidade Federal do Vale do São Francisco, Petrolina, Pernambuco, Brasil. \\ Daniel Vicentini de Oliveira \\ Universidade Estadual de Campinas, Campinas, São Paulo, Brasil. \\ Caio Rosas Moreira \\ Universidade Estadual de Maringá, Maringá, Paraná, Brasil. \\ Lenamar Fiorese Vieira \\ Universidade Estadual de Maringá, Maringá, Paraná, Brasil.
}

\section{Resumo}

Este estudo analisou o nível de motivação acadêmica e percepção de competência profissional de acadêmicos de Educação Física. Participaram 191 acadêmicos que responderam as Escalas de Motivação Acadêmica e de Autopercepção de Competência Profissional em Educação Física e Desportos. As mulheres apresentaram maior Regulação Integrada. Os alunos que não realizam estágio demonstraram maior Desmotivação, enquanto os que realizam estágio apresentaram maior Regulação Integrada e competência profissional. Ainda, a percepção de competência profissional de quem não realiza estágio se correlacionou principalmente com a Regulação Externa e a Desmotivação. Concluiu-se que o estágio promove maior percepção de competência profissional e melhores níveis de motivação autodeterminada.

Palavras-chave: Autodeterminação. Habilidade. Conhecimento. Estudantes.

\section{Introdução}

O curso presencial de graduação em Educação Física está entre os 20 cursos da rede privada com maior índice de concluintes, segundo o Instituto Nacional de Estudos e Pesquisas Educacionais (CAPELATO, 2015), sendo que 46,2\% dos ingressantes finalizam a graduação. Com este grande número de profissionais entrando no mercado de trabalho, é fundamental o profissional apresentar competência profissional para que seja bem-sucedido (LEMOS; LEE; ROSE JÚNIOR, 2010). Este perfil de comportamento é necessário, uma vez que gestores da área da Educação Física demandam profissionais com competências não só relacionadas ao conhecimento teórico e técnico, mas também com um perfil proativo e habilidades relaciona- 
das ao marketing e à gestão (ANVERSA; OLIVEIRA, 2011). Assim, a percepção de competência do profissional se inicia durante a graduação, ao passo que as experiências ao longo deste processo favorecem o fortalecimento dessa competência, elevando a motivação para as tarefas a serem realizadas (BATISTA; MATOS; GRAÇA, 2011).

A Teoria da Autodeterminação (TAD) é uma macroteoria que aborda amplamente a motivação e a personalidade humana (DECI; RYAN, 2012) e tem sido a base teórica mais utilizada para investigar os aspectos motivacionais nos mais diversos contextos da vida humana (DECI; RYAN, 2008). Destacam-se investigações nos campos da educação (KORTHAGEN; EVELEIN, 2016), do trabalho (OLAFSEN et. al., 2015), do suporte parental (MAGEAU et al., 2016), da sustentabilidade (AITKEN; PELLETIER; BAXTER, 2016), do esporte (CARPENTIER; MAGEAU, 2016) e dos cuidados com a saúde (LOPEZ et al., 2016), demonstrando a relação da motivação autodeterminada como fator-chave para o desenvolvimento humano.

Diferentes facetas da motivação e da personalidade humana são abordadas por meio das seis microteorias da TAD (DECI; RYAN, 2012), sendo estas: I. Teoria de avaliação cognitiva: aborda a motivação intrínseca e os efeitos dos contextos sociais sob a motivação; II. Teoria da orientação organísmica: aborda as diferentes formas de motivação extrínseca e seu continuum de internalização; III. Teoria das orientações de causalidade: onde o sujeito pode ser orientado de forma impessoal, controlada ou autônoma; IV. Teoria das três necessidades psicológicas básicas: postula que o bem-estar psicológico depende da autonomia, da competência e das relações sociais; V. Teoria dos conteúdos de meta: aborda as distinções entre metas intrínsecas ou extrínsecas e seu impacto na motivação e no bem-estar; VI: Teoria da motivação de relacionamentos: postula que a qualidade de relacionamentos é essencial para o bem-estar do sujeito.

Ao passo que a motivação pode ser regulada por um continuum, a TAD aponta seis regulações de motivação (DECI; RYAN, 2012), variando desde a ausência de interesse pela atividade até a realização pelo simples prazer e satisfação nesta tarefa. A desmotivação é caracterizada pela ausência de motivação. A regulação externa é a forma mais simples da motivação extrínseca, em que o indivíduo age para obter recompensas externas ou evitar punições. A regulação introjetada é o comportamento controlado por pressões internas causadas por influências extrínsecas. A regulação identificada ocorre quando há percepção de que o comportamento é importante para a sua vida, a regulação integrada é a forma mais plena de internalização, pois integra a identificação com outros aspectos da sua identidade. E a regulação intrínseca é considerada o estilo de autodeterminação perfeito, no qual as atividades são realizadas simplesmente pela satisfação ao se realizar (TAYLOR, 2015; VANSTEENKISTE; RYAN, 2013).

Estudos recentes têm aplicado a TAD em diversos contextos relacionados à Educação Física, tais como esportivo (MOREIRA et al., 2016; CURRAN; HILL; NIEMIEC, 2013), escolar (PIZANI et al., 2016; STANDAGE et al., 2012), universitário (COSTA et al., 2015) e na prática de exercícios físicos (MEURER; BENEDETTI; MAZO, 2012; SEBIRE et al., 2013). Pesquisas também têm verificado a percepção de competência profissional na Educação Física relacionada a variáveis como as preocupações pedagógicas (RIBEIRO et al., 2015), os diferentes momentos da graduação (LEMOS; LEE; ROSE JÚNIOR, 2010), a realização de estágio (VIEIRA; VIEIRA; FERNANDES, 2006) e ao longo da carreira docente (FARIAS et al., 2012). No entanto, não foram encontrados estudos que relacionem a percepção de competência profissional de acadêmicos de Educação Física à motivação acadêmica, sendo esta a lacuna que o presente estudo visa preencher. Dessa forma, esta pesquisa torna-se relevante na medida em que poderá contribuir com o trabalho de docentes e profissionais envolvidos na formação acadêmica dos futuros profissionais de Educação Física. 
Diante das considerações expostas anteriormente, o objetivo do presente estudo foi analisar o nível de motivação acadêmica e percepção de competência profissional de estudantes do curso de Educação Física de uma universidade no estado do Paraná, buscando especificamente comparar as variáveis em função do sexo, do turno das aulas e da realização ou não de estágio na área, além de verificar a correlação entre as dimensões das duas variáveis.

\section{Métodos}

\section{Participantes}

Foram convidados a participar deste estudo descritivo todos os acadêmicos do curso de Educação Física de uma Instituição de Ensino Superior (IES) privada do noroeste do estado do Paraná, totalizando aproximadamente 300 alunos. No entanto, aceitaram participar voluntariamente do estudo 191 acadêmicos de ambos os sexos (105 homens e 86 mulheres), com média de idade de 24,80 $\pm 5,76$ anos, sendo que $106(55,5 \%)$ eram do período matutino e $85(44,5 \%)$ do noturno. Destaca-se que 55\% dos acadêmicos já realizavam estágio na área da Educação Física, enquanto $45 \%$ apenas estudavam.

\section{Instrumentos}

Para avaliar o nível de motivação dos estudantes foi utilizada a Escala de Motivação Acadêmica (EMA), elaborada por Vallerand et al. (1989) e validada para o contexto brasileiro por Guimarães e Bzuneck (2008). A escala consiste em 31 questões em uma escala do tipo likert que varia de 1 (nenhuma correspondência) a 7 (total correspondência) e que visam responder à pergunta "Por que venho à universidade?". Os resultados são obtidos por meio da média dos itens, que se distribuem em sete domínios baseados no continuum da TAD (Desmotivação, Regulação Externa, Regulação Externa Social, Regulação Introjetada, Regulação Identificada, Regulação Integrada e Motivação Intrínseca). O alfa de Conbrach das dimensões deste instrumento variou de $\alpha=0,55$ a $\alpha=0,79$, indicando moderada para forte confiabilidade dos dados.

Para verificar a competência profissional percebida foi utilizada a Escala de Autopercepção de Competência Profissional em Educação Física e Desportos (NASCIMENTO, 1999). Esse instrumento é composto por 30 itens em uma escala do tipo likert de 0 (Nenhum domínio) a 5 (Domínio total). As questões são divididas em duas dimensões e nove indicadores: 1. Conhecimento Profissional (Conhecimento Disciplinar, Conhecimento Pedagógico e Conhecimento do Contexto) e 2. Habilidades Profissionais (Planejamento, Comunicação, Avaliação, Organização e Gestão, Incentivação e Autorreflexão). O resultado é obtido por meio da média de cada indicador e da média geral de cada dimensão. $\mathrm{O}$ alfa de Conbrach dos indicadores e dimensões deste instrumento variou de $\alpha=0,57$ a $\alpha=0,97$, indicando moderada para forte confiabilidade dos dados.

\section{Procedimentos}

O estudo está integrado ao projeto institucional aprovado pelo comitê de ética da Universidade Estadual de Maringá, sob parecer $n^{\circ}$ 238/2011. Inicialmente, foi realizado um contato com a direção da IES e com a coordenação do curso de Educação Física, solicitando autorização para realizar uma coleta de dados com os acadêmicos. Participaram do estudo os acadêmicos que concordaram com a pesquisa e assinaram o Termo de Consentimento Livre e Esclarecido. A coleta de dados foi realizada no segundo semestre de 2015, e os acadêmicos responderam individualmente ao questionário, levando em média 30 minutos. 
Análise dos dados

Para a análise da distribuição dos dados, utilizou-se o teste de Kolmogorov-Smirnov. Os dados não apresentaram distribuição normal, assim foram utilizados Mediana (Md) e Quartis (Q1; Q3) para caracterização dos resultados. Para a avaliação da consistência interna das dimensões dos questionários de motivação acadêmica e de autopercepção de competência profissional, efetuou-se o alfa de Cronbach. Na comparação entre os grupos, foi utilizado o teste "U" de Mann-Whitney. Calculou-se também o tamanho do efeito $(d)$ por meio do modelo proposto por Cohen (1992) para diferenças dos valores de dois grupos independentes. De acordo com os critérios de Cohen, um valor $d=0,20$ representa tamanho de efeito pequeno; $d$ $=0,50$, médio; e $d=0,80$, grande. Para verificar a correlação entre as variáveis, efetuou-se o coeficiente de correlação de Spearman. A significância adotada foi de $p<0,05$. Os dados foram analisados no software SPSS 22.0.

\section{Resultados}

Verificou-se (Tabela 1) que os acadêmicos de Educação Física de ambos os sexos demonstraram níveis de motivação similares, com baixo nível de desmotivação e maiores escores nas regulações mais próximas da motivação intrínseca. Houve diferença significativa apenas na regulação integrada $(\mathrm{p}=0,010)$, em que as mulheres apresentaram maior escore, indicando que as estudantes estão mais próximas da motivação intrínseca do que os homens. No entanto, tal efeito pode ser considerado pequeno $(d<0,50)$. Na percepção de competência profissional, observouse que os escores apresentaram níveis moderados, não apresentando nenhuma diferença significativa entre os grupos $(\mathrm{p}<0,05)$.

Tabela 1 - Comparação das regulações de motivação acadêmica e percepção de competência profissional dos acadêmicos de Educação Física em função do sexo.

\begin{tabular}{|c|c|c|c|}
\hline \multirow[t]{2}{*}{ VARIÁVEIS } & $\begin{array}{l}\text { Masculino } \\
(\mathrm{n}=105)\end{array}$ & $\begin{array}{l}\text { Feminino } \\
(\mathbf{n}=86)\end{array}$ & \multirow{2}{*}{$\begin{array}{l}\text { Tam } \\
\text { do e }\end{array}$} \\
\hline & Md (Q1; Q3) & Md (Q1; Q3) & \\
\hline \multicolumn{4}{|l|}{$\begin{array}{l}\text { MOTIVAÇÃO ACADÊMI- } \\
\text { CA }\end{array}$} \\
\hline Desmotivação & $1,33(1,00 ; 1,33)$ & $1,33(1,00 ; 1,87) 0,296$ & 0,14 \\
\hline Regulação Externa & $3,40(2,40 ; 4,80)$ & $3,50(2,40 ; 4,80) 0,975$ & $-0,02$ \\
\hline Regulação Externa (Social) & $1,00(1,00 ; 2,00)$ & $1,50(1,00 ; 2,00) 0,291$ & 0,01 \\
\hline Regulação Introjetada & $4,20(3,20 ; 5,20)$ & $4,60(3,70 ; 5,40) 0,158$ & -0.07 \\
\hline Regulação Identificada & $5,50(4,00 ; 6,00)$ & $5,25(4,50 ; 6,12) 0,704$ & -0.03 \\
\hline Regulação Integrada & $5,75(4,87 ; 6,37)$ & $6,25(5,50 ; 6,75) \mathbf{0 , 0 1 0} *$ & -0.40 \\
\hline Regulação Intrínseca & $4,67(3,67 ; 5,67)$ & $4,67(3,67 ; 5,67) 0588$ & -0.01 \\
\hline \multicolumn{4}{|c|}{ COMPETÊNCIA PROFISSIONAL } \\
\hline Conhecimento profissional & $3,29(2,57 ; 3,71)$ & $3,10(2,47 ; 3,61) 0,152$ & 0,20 \\
\hline Conhecimento disciplinar & $3,28(2,71 ; 3,85)$ & $3,07(2,53 ; 3,71) 0,299$ & 0,15 \\
\hline Conhecimento pedagógico & $3,25(2,50 ; 3,75)$ & $3,00(2,50 ; 3,50) 0,196$ & 0,16 \\
\hline Conhecimento do contexto & $3,00(2,66 ; 3,66)$ & $3,00(2,33 ; 3,41) 0,088$ & 0,27 \\
\hline Habilidades profissionais & $3,41(2,77 ; 3,81)$ & $3,18(2,61 ; 3,80) 0,285$ & 0,15 \\
\hline Planejamento & $3,50(3,00 ; 4,00)$ & $3,50(2,87 ; 4,50) 0,836$ & 0,02 \\
\hline Comunicação & $3,50(2,50 ; 4,00)$ & $3,00(2,33 ; 3,66) 0,172$ & 0,14 \\
\hline Avaliação & $3,33(2,66 ; 3,66)$ & $3,00(2,33 ; 3,66) 0,726$ & 0,07 \\
\hline
\end{tabular}




$\begin{array}{llll}\text { Organização e gestão } & 3,50(3,00 ; 4,00) & 3,50(2,75 ; 3,81) 0,509 & 0,11 \\ \text { Incentivação } & 3,33(2,66 ; 4,00) & 3,33(2,33 ; 4,00) 0,256 & 0,18 \\ \text { Autorreflexão } & 3,00(3,00 ; 4,00) & 3,00(2,50 ; 3,62) 0,123 & 0,22\end{array}$

$*$ Diferença significativa $-\mathrm{p}<0,05$.

Fonte: Os autores, 2017

A Tabela 2 apresenta a comparação das variáreis em função do turno do curso. Os acadêmicos apresentaram medianas com escores moderados tanto nas regulações de motivação acadêmica quanto na autopercepção de competência profissional. Não houve diferença significativa $(\mathrm{p}<0,05)$ em nenhuma das variáveis, demonstrando que o horário de realização das aulas não é um fator interveniente na motivação e na competência profissional dos alunos.

Tabela 2 - Comparação das regulações de motivação acadêmica e percepção de competência profissional dos acadêmicos de Educação Física em função do turno.

\begin{tabular}{|c|c|c|c|c|}
\hline \multirow[t]{2}{*}{ VARIÁVEIS } & $\begin{array}{l}\text { Matutino } \\
(\mathrm{n}=106)\end{array}$ & $\begin{array}{l}\text { Noturno } \\
(\mathrm{n}=85)\end{array}$ & \multirow[t]{2}{*}{$\mathbf{P}$} & \multirow{2}{*}{$\begin{array}{l}\text { Tamanho } \\
\text { do efeito }\end{array}$} \\
\hline & Md (Q1; Q3) & Md (Q1; Q3) & & \\
\hline \multicolumn{5}{|l|}{$\begin{array}{l}\text { MOTIVAÇÃO ACADÊMI- } \\
\text { CA }\end{array}$} \\
\hline Desmotivação & $1,33(1,00 ; 2,17)$ & $1,33(1,00 ; 2,00)$ & 0,972 & $-0,01$ \\
\hline Regulação Externa & $3,30(2,35 ; 5,00)$ & $3,60(2,50 ; 4,60)$ & 0,998 & $-0,01$ \\
\hline Regulação Externa (Social) & $1,12(1,00 ; 2,00)$ & $1,25(1,00 ; 2,00)$ & 0,508 & $-0,09$ \\
\hline Regulação Introjetada & $4,40(3,20 ; 5,40)$ & $4,40(3,30 ; 5,20)$ & 0,737 & $-0,08$ \\
\hline Regulação Identificada & $5,50(4,50 ; 6,00)$ & $5,00(4,00 ; 6,00)$ & 0,407 & 0,11 \\
\hline Regulação Integrada & $6,00(5,00 ; 6,50)$ & $5,75(5,00 ; 6,75)$ & 0,914 & 0,01 \\
\hline Regulação Intrínseca & $4,67(3,67 ; 5,67)$ & $4,67(4,00 ; 5,67)$ & 0,803 & $-0,05$ \\
\hline \multicolumn{5}{|c|}{ COMPETÊNCIA PROFISSIONAL } \\
\hline Conhecimento profissional & $3,12(2,55 ; 3,66)$ & $3,19(2,51 ; 3,61)$ & 0,745 & 0,14 \\
\hline Conhecimento disciplinar & $3,14(2,71 ; 3,85)$ & $3,28(2,57 ; 3,71)$ & 0,999 & 0,08 \\
\hline Conhecimento pedagógico & $3,00(2,50 ; 3,75)$ & $3,25(2,50 ; 3,50)$ & 0,900 & 0,06 \\
\hline Conhecimento do contexto & $3,00(2,33 ; 3,66)$ & $3,00(2,33 ; 3,66)$ & 0,437 & 0,21 \\
\hline Habilidades profissionais & $3,31(2,75 ; 3,85)$ & $3,33(2,66 ; 3,80)$ & 0,678 & 0,18 \\
\hline Planejamento & $3,50(3,00 ; 4,00)$ & $3,50(2,75 ; 4,00)$ & 0,621 & 0,14 \\
\hline Comunicação & $3,00(2,50 ; 4,00)$ & $3,00(2,50 ; 4,00)$ & 0,826 & 0,12 \\
\hline Avaliação & $3,00(2,66 ; 3,66)$ & $3,33(2,33 ; 3,66)$ & 0,829 & 0,13 \\
\hline Organização e gestão & $3,50(3,00 ; 4,00)$ & $3,50(2,75 ; 3,75)$ & 0,302 & 0,26 \\
\hline Incentivação & $3,33(2,66 ; 4,00)$ & $3,33(2,50 ; 3,83)$ & 0,319 & 0,22 \\
\hline Autorreflexão & $3,00(2,50 ; 4,00)$ & $3,50(2,50 ; 4,00)$ & 0,749 & 0,05 \\
\hline
\end{tabular}

$*$ Diferença significativa $-\mathrm{p}<0,05$.

Fonte: Os autores, 2017

Pode-se observar na Tabela 3 a comparação do nível de motivação acadêmica e percepção de competência profissional dos acadêmicos em função da realização ou não de estágio na área. Os acadêmicos que não realizam estágio apresentaram maior escore na regulação de Desmotivação $(\mathrm{p}=0,012)$, enquanto os alunos que já realizam estágio apresentaram escore 
superior na Regulação Integrada $(\mathrm{p}=0,027)$. Contudo, a análise do tamanho do efeito demonstrou magnitude de efeito pequena.

Tabela 3 - Comparação das regulações de motivação acadêmica e percepção de competência profissional dos acadêmicos de Educação Física em função da realização ou não de estágio na área.

\begin{tabular}{|c|c|c|c|c|}
\hline \multirow[t]{2}{*}{ VARIÁVEIS } & $\begin{array}{l}\text { Realiza estágio } \\
(\mathrm{n}=105)\end{array}$ & $\begin{array}{l}\text { Não realiza } \\
(n=86)\end{array}$ & \multirow[t]{2}{*}{$\mathbf{P}$} & \multirow[t]{2}{*}{$\begin{array}{l}\text { Tamanho } \\
\text { do efeito }\end{array}$} \\
\hline & Md (Q1; Q3) & Md (Q1; Q3) & & \\
\hline \multicolumn{5}{|l|}{$\begin{array}{l}\text { MOTIVAÇÃO ACADÊ- } \\
\text { MICA }\end{array}$} \\
\hline Desmotivação & $1,17(1,00 ; 1,91)$ & $1,50(1,00 ; 2,50)$ & $\mathbf{0 , 0 1 2 *}$ & $-0,47$ \\
\hline Regulação Externa & $3,40(2,40 ; 4,80)$ & $3,40(2,40 ; 4,80)$ & 0,760 & 0,01 \\
\hline Regulação Externa (Social) & $1,25(1,00 ; 1,87)$ & $1,25(1,00 ; 2,25)$ & 0,456 & $-0,22$ \\
\hline Regulação Introjetada & $4,60(3,20 ; 5,40)$ & $4,30(3,35 ; 5,00)$ & 0,257 & 0,10 \\
\hline Regulação Identificada & $5,50(4,25 ; 6,25)$ & $5,50(4,00 ; 6,00)$ & 0,695 & 0,06 \\
\hline Regulação Integrada & $6,00(5,37 ; 6,62)$ & $5,75(4,75 ; 6,50)$ & $\mathbf{0 , 0 2 7 *}$ & 0,33 \\
\hline Regulação Intrínseca & $4,67(3,67 ; 5,67)$ & $4,67(3,67 ; 5,41)$ & 0,253 & 0,17 \\
\hline \multicolumn{5}{|c|}{ COMPETÊNCIA PROFISSIONAL } \\
\hline Conhecimento profissional & $3,32(2,62 ; 3,79)$ & $3,02(2,42 ; 3,41)$ & $\mathbf{0 , 0 0 3 *}$ & 0,49 \\
\hline Conhecimento disciplinar & $3,42(2,71 ; 3,85)$ & $3,14(2,42 ; 3,57)$ & $\mathbf{0 , 0 2 1} *$ & 0,36 \\
\hline Conhecimento pedagógico & $3,25(2,75 ; 3,87)$ & $3,00(2,50 ; 3,50)$ & $0,001 *$ & 0,49 \\
\hline Conhecimento do contexto & $3,33(2,66 ; 3,66)$ & $3,00(2,33 ; 3,33)$ & $0,001 *$ & 0,48 \\
\hline Habilidades profissionais & $3,44(2,82 ; 3,93)$ & $3,16(2,43 ; 3,61)$ & $0,006 *$ & 0,46 \\
\hline Planejamento & $3,50(3,00 ; 4,50)$ & $3,50(2,50 ; 4,00)$ & $\mathbf{0 , 0 1 5 *}$ & 0,38 \\
\hline Comunicação & $3,50(2,50 ; 4,00)$ & $3,00(2,50 ; 4,00)$ & $\mathbf{0 , 0 1 2 *}$ & 0,38 \\
\hline Avaliação & $3,33(2,66 ; 3,66)$ & $3,00(2,33 ; 3,66)$ & $0,016 *$ & 0,41 \\
\hline Organização e gestão & $3,50(3,00 ; 4,12)$ & $3,25(2,75 ; 3,75)$ & $0,001 *$ & 0,48 \\
\hline Incentivação & $3,33(2,66 ; 4,00)$ & $3,00(2,33 ; 3,66)$ & $\mathbf{0 , 0 2 1} *$ & 0,33 \\
\hline Autorreflexão & $3,50(3,00 ; 4,00)$ & $3,00(2,50 ; 3,50)$ & $\mathbf{0 , 0 2 6 *}$ & 0,34 \\
\hline
\end{tabular}

$*$ Diferença significativa $-\mathrm{p}<0,05$.

Fonte: Os autores, 2017

Em relação à competência profissional (Tabela 3), houve diferença significativa em todas as dimensões e indicadores $(\mathrm{p}<0,05)$, demonstrando que a realização de estágio proporciona maior percepção de competência em relação ao conhecimento e às habilidades profissionais aos alunos. No entanto, a análise do tamanho do efeito demonstrou magnitude de efeito pequena $(d<0,50)$.

Ao analisar a correlação entre as regulações de motivação acadêmica e as dimensões/indicadores de competência profissional, verificou-se correlação significativa apenas entre o indicador de Avaliação e a Regulação Introjetada $(r=0,20)$. Considerando que a realização de estágio na área demonstrou ser um elemento interveniente nas variáveis, optou-se por verificar a correlação entre as variáveis dentro de cada grupo. Nos acadêmicos que não realizavam estágio foram encontradas as seguintes correlações significativas $(p<0,05)$ : Desmotivação e Conhecimento Disciplinar ( $\mathrm{r}=0,19)$; Desmotivação e Conhecimento do Contexto $(\mathrm{r}=0,24)$; Desmotivação e Conhecimento Profissional ( $\mathrm{r}=0,20)$; Desmotivação e Avaliação $(\mathrm{r}=0,17)$; Desmotivação e Incentivação $(\mathrm{r}=0,17)$; Regulação Externa e Conhecimento do 
Contexto (r=0,20); Regulação Externa e Conhecimento Profissional (r=0,17); Regulação Externa e Incentivação $(r=0,26)$; Incentivação e Autorreflexão $(r=0,19)$; Regulação Introjetada e Conhecimento Pedagógico ( $\mathrm{r}=0,19)$; Regulação Intrínseca e Conhecimento Pedagógico $(\mathrm{r}=0,21)$; Regulação Intrínseca e Conhecimento Profissional $(\mathrm{r}=0,17)$. Já entre os alunos que realizavam estágio, houve correlação significativa apenas entre o indicador de Avaliação e a Regulação Introjetada $(\mathrm{r}=0,15)$.

\section{Discussão}

Considerando-se a ausência de estudos que relacionem a percepção de competência profissional à motivação acadêmica em estudantes universitários, especificamente na área da Educação Física, o presente estudo avança ao identificar como o sexo, o turno do curso e a realização de estágio interferem nessas variáveis. De modo geral, os alunos apresentaram baixas Desmotivação e Regulação Externa (social), sendo mais motivados pela Regulação Integrada, que já pode ser considerada uma forma de motivação intrínseca (DECI; RYAN, 2012). Subsequentemente, os acadêmicos demonstraram uma percepção de competência considerada suficiente (NASCIMENTO, 1999), podendo-se destacar a realização de estágio como fatorchave para uma maior percepção.

Um dos principais achados do presente estudo foi a análise da atuação na área (realização de estágio não obrigatório) como um fator interveniente na motivação acadêmica e na percepção de competência profissional (Tabela 3). Os estudantes que tinham a experiência do estágio apresentaram uma maior percepção de competência em todas as dimensões e indicadores. Tal resultado pode estar relacionado à microteoria das necessidades psicológicas básicas, na qual a competência está inclusa como um fator para o bem-estar dos sujeitos (RYAN et al., 2008). Ao estagiar na área, os estudantes vivenciam certa autonomia para desenvolver suas atividades, adquirem competência para desempenhar suas funções e mantêm relações sociais com seus alunos. Desse modo, Markland et al. (2005) destacam que, quanto mais as pessoas estiverem engajadas voluntariamente e com alto grau de disposição para agir, mais estarão aptas para aprender e aplicar novas estratégias e competências.

Silva Junior et al. (2016) ressaltam a importância da relação constante entre teoria e prática por meio do estágio curricular, uma vez que o estágio proporciona ao futuro professor experiências e até mesmo possíveis conflitos que poderão enfrentar no ambiente de atuação profissional. Neste contexto, o estágio não obrigatório deve ser algo muito mais incentivado dentro das IES, por se tratar de uma vivência que possibilita um grande avanço para a formação acadêmica e preparação para o mercado de trabalho. Mercado este que exige determinadas características para um bom profissional de Educação Física ser contratado, tais como domínio sobre conhecimentos técnicos, bom relacionamento interpessoal com as pessoas, perfil proativo, noção de administração e marketing pessoal (ANVERSA; OLIVEIRA, 2011). Assim, o estágio pode ser uma experiência de grande impacto para a vida profissional de um acadêmico, tendo em vista que possibilita maior contato com a realidade do mercado de trabalho e maior atuação prática durante sua formação acadêmica (MOLETTA et al., 2013; SANTOS et al., 2013), proporcionando maior percepção de competência (Tabela 3) para sua futura atuação.

Em se tratando da motivação, ressalta-se que realizar alguma atividade prática na área ao longo do curso (COSTA et al., 2015) é uma importante ferramenta para evitar a ausência de motivação acadêmica (LOPES et al., 2015). Ainda, esta experiência favorece a internalização da importância dos estudos na formação profissional, uma vez que a internalização deste comportamento integra a identificação com outros aspectos da vida do aluno (DECI; RYAN, 2000). 
De acordo com a microteoria da avaliação cognitiva (DECI; RYAN, 2008), a motivação intrínseca sofre grande efeito dos contextos sociais em que o indivíduo está inserido (VANSTEENKISTE; RYAN, 2013). O resultado desta pesquisa corrobora a teoria, tendo em vista que o contexto social do estágio não obrigatório pode ser considerado um elemento interveniente na motivação intrínseca dos acadêmicos. Além disso, vai ao encontro da microteoria das orientações de causalidade (DECI; RYAN, 2008), na qual os comportamentos influenciados por recompensas externas (controlados) tendem a diminuir a motivação intrínseca, enquanto agir por escolha (comportamento autônomo) tende a aumentá-la, além de melhorar a saúde psicológica (DECI; RYAN, 2000), o que é o caso dos acadêmicos deste estudo, por se tratar de um estágio não obrigatório.

Resultados semelhantes foram encontrados por Costa et al. (2015) em estudo com acadêmicos do curso de Educação Física de uma IES do estado do Paraná. Nesta pesquisa, a Desmotivação demonstrou os menores escores, enquanto a Regulação Integrada foi a mais destacada. Isto evidencia que os alunos compreendem a necessidade e a importância das suas atitudes e ressalta a proximidade dessa regulação à motivação autodeterminada.

Ao correlacionar de maneira geral a motivação acadêmica com a percepção de competência profissional, apenas a Avaliação se associou com a Regulação Introjetada, também observado para o grupo que realiza estágio. Isto pode ser decorrente da experiência que estas pessoas já têm ou que sabem que devem ter e que se tornam importantes por serem exigências externas impostas pelo mercado de trabalho do profissional de Educação Física (ANVERSA; OLIVEIRA, 2011; DECI; RYAN, 2012).

Ainda, para o grupo específico de quem não realiza estágio, a percepção de competência profissional esteve mais correlacionada com a Regulação Externa e com a Desmotivação do que com os outros tipos de motivação. De acordo com a microteoria das necessidades psicológicas básicas (DECI; RYAN, 2012), pessoas que demonstram baixos níveis de competência em uma determinada atividade tendem a se desmotivar ao longo do tempo. Costa et al. (2015) verificaram que alunos do último ano do curso de Educação Física são mais desmotivados com relação aos do primeiro ano, o que pode estar atrelado a uma preocupação com a entrada no mercado de trabalho. No presente estudo, a ausência de experiências práticas vivenciadas durante a graduação pode ser um agravante tanto para a motivação ao longo deste processo, como também pela falta de percepção de competência profissional que estes acadêmicos poderão apresentar quando estiverem iniciando no mercado de trabalho.

O sexo (Tabela 1) demonstrou ser um fator interveniente somente na Regulação Integrada, a mais próxima da Regulação Intrínseca e mais completa forma de internalização (DECI; RYAN, 2000), uma vez que as mulheres demonstraram um comportamento que surge da vontade e da valorização da atividade maior do que os homens. No entanto, a competência profissional não apresentou diferenças significativas, corroborando os estudos de Ribeiro et al. (2015) e Lemos, Lee e Rose Júnior (2010), nos quais o sexo não foi um fator interveniente. Estudar pela manhã ou pela noite não influenciou a motivação acadêmica e a percepção de competência profissional (Tabela 2), corroborando o estudo de Vieira, Vieira e Fernandes (2006), no qual o turno também não foi um fator interveniente na percepção de competência profissional de 111 acadêmicos de Educação Física da Universidade Estadual de Maringá dos períodos integral e noturno.

Sendo assim, embora o presente estudo forneça importantes evidências empíricas sobre a importância da motivação intrínseca e uma percepção de competência positiva de acadêmicos, algumas limitações precisam ser apontadas. Inicialmente, apenas estudantes de uma única IES do estado do Paraná foram avaliados, o que não representa a realidade do ensino superior do país. Em segundo lugar, só foram analisados os estudantes de um mesmo curso (Educação Física), indicando que não há garantia de que os resultados aqui apresentados sejam semelhantes em cursos de outras áreas do conhecimento. Em terceiro lugar, o estudo se 
restringiu a avaliar apenas um momento durante toda a formação acadêmica dos alunos, não podendo determinar se a evolução do aluno durante a graduação pode ser fator que influencie na motivação e na percepção de competência dos acadêmicos. Assim, sugere-se a utilização de um design longitudinal para a avaliação da motivação acadêmica e da percepção de competência dos estudantes durante o processo de formação universitária.

\title{
Conclusão
}

Conclui-se que a experiência com o estágio não obrigatório pode ser considerada um fator determinante para promover melhor percepção de competência profissional, assim como melhores níveis de motivação acadêmica ao longo da graduação em Educação Física. Ressalta-se que, para aqueles que não realizam estágio, a baixa percepção de competência se associa com a menor motivação dos alunos. Como implicações práticas destes resultados, pode-se considerar o maior incentivo por parte tanto das IES como dos professores ao longo de toda a graduação, indicando a importância para a preparação e a experiência que um estágio pode possibilitar para uma melhor formação acadêmica e profissional do aluno.

\section{ACADEMIC MOTIVATION AND PERCEPTION OF PROFESSIONAL COMPE- TENCE OF ACADEMICS OF PHYSICALEDUCATION}

\begin{abstract}
This study analyzed the academic motivation and perception of professional competence levels among Physical Education students. The subjects were 191 academics who answered the Academic Motivation Scale and the Self-Perception Scale of Professional Competence in Physical Education and Sports. Women presented greater Integrated Regulation. The students who did not perform the internship had higher demotivation, while those who completed the internship had higher Integrated Regulation and professional competence. Further, the perception of professional competence of those who do not perform internship has correlated mainly with the External Regulation and Desmotivation. It was concluded that internship promotes greater perception of professional competence and better levels of self-determined motivation. Keywords: Self-determination. Aptitude. Knowledge. Students.
\end{abstract}

\section{MOTIVACIÓN ACADÉMICA Y PERCEPCIÓN DE LA COMPETENCIA PROFE- SIONAL DE LOS ACADEMICOS DE EDUCACIÓN FÍSICA}

\begin{abstract}
Resumen
En este estudio se analizó el nivel de motivación académica y la percepción de la competencia profesional de los estudiantes de Educación Física. Participaron 191 estudiantes que respondieron a la Escala de Motivación académica y la Escala de Autopercepción de la competencia profesional en Educación Física y Deportes. Las mujeres mostraron una mayor regulación integrada. Los estudiantes que no realizan practicas tuvieron mayor desmotivación, mientras que aquellos que realizan practicas mostraron mayor regulación integrada y competencia profesional. Sin embargo, la percepción de la competencia profesional de los estudiantes que no realizan practicas se asoció principalmente al regulación externa y desmotivación. Se concluyó que la practicas promueve una mayor conciencia de la competencia profesional y mejores niveles de motivación autodeterminada.
\end{abstract}

Palabras clave: Autodeterminación. Aptitud. Conocimiento. Estudiantes. 


\section{Referências}

AITKEN, N. M.; PELLETIER, L. G.; BAXTER, D. E. Doing the difficult stuff: influence of self-determined motivation toward the environment on transportation proenvironmental behavior. Ecopsychology, v. 8, n. 2, p. 153-162, jun. 2016.

ANVERSA, A. L. B.; OLIVEIRA, A. A. B. Personal trainer: competências profissionais demandadas pelo mercado de trabalho. Pensar a prática, v. 14, n. 3, p. 1-17, set./dez. 2011.

BATISTA, P.; MATOS, Z.; GRAÇA, A. A autopercepção das competências profissionais do desporto: efeito da área de intervenção e da experiência profissional. Revista de Ciencias del Deporte, v. 7, p. 117-131, 2011.

CAPELATO, R. Mapa do ensino superior no Brasil. Convergência, 2015.

CARPENTIER, J.; MAGEAU, G. A. Predicting sport experience during training: the role of change-oriented feedback in athletes' motivation, self-confidence and needs satisfaction fluctuations. Journal of Sport \& Exercise Psychology, v. 38, n. 1, p. 45-58, fev. 2016.

COHEN, J. Quantitative methods in psychology: a power primer. Psychological Bulletin, v.112, n.1, p.155-159, 1992.

COSTA, L. A.; NASCIMENTO JÚNIOR, J. R. A.; MEDEIROS, A. I. A.; VIEIRA, L. F. Estudo dos fatores motivacionais e orientação de vida de universitários. Cinergis, v. 16, n. 2, p. 136-141, abr./jun. 2015.

CURRAN, T.; HILL, A. P.; NIEMIEC, C. P. A conditional process model of children's behavioral engagement and behavioral disaffection in sport based on self-determination theory. Journal of Sport and Exercise Psychology, v. 35, n. 1, p. 30-43, fev. 2013.

DECI, E. L.; RYAN, R. M. Self-determination theory. In: LANGE, P. A. M. V.; KRUGLANSKI, A. W.; HIGGINS, E. T. Handbook of theories of social psychology. Thousand Oaks: Sage, 2012, v. 1. p. 416-437.

DECI, E.L.; RYAN, R. M. Self-determination theory: a macrotheory of human motivation, development, and health. Can Psychol, v. 49, n. 3, p.182-185, ago. 2008.

DECI, E.L.; RYAN, R.M. The "what" and "why" of goal pursuits: human needs and selfdetermination of behavior. Psychological Inquiry, v. 11, n. 4, p. 227-268, 2000.

FARIAS, G. O.; NASCIMENTO, J. V.; GRAÇA, A.; BATISTA, P. M. F. Competências profissionais em Educação Física: uma abordagem ao longo da carreira docente. Motriz, Rio Claro, v. 18, n. 4, p. 656-666, out./dez. 2012.

GUIMARÃES, S. É. R.; BZUNECK, J. A. Propriedades psicométricas de um instrumento para avaliação da motivação de universitários. Ciências \& Cognição, v. 13, n. 1, p. 101-113, mar. 2008. 
KORTHAGEN, F. A. J.; EVELEIN, F. G. Relations between student teachers' basic needs fulfillment and their teaching behavior. Teaching and Teacher Education, v. 60, p. 234-244, 2016.

LEMOS, E. D.; LEE, C. L; ROSE JÚNIOR, D. Autopercepção de competência profissional dos estudantes de um curso superior de atividade física. Revista Corpoconsciência, Santo André, v. 14, n. 2, p. 30-41, jul./dez. 2010.

LOPES, L. M. S.; PINHEIRO, F. M. G.; SILVA, A. C. R.; ABREU, E. S. Aspectos da motivação intrínseca e extrínseca: uma análise com discentes de Ciências Contábeis da Bahia na perspectiva da Teoria da Autodeterminação. Revista de Gestão, Finanças e Contabilidade, v. 5, n. 1, p. 21, 2015.

LOPEZ, R. B.; MILYAVSKAYA, M.; HOFMANN, W.; HEATHERTON, T. F. Motivational and neural correlates of self-control of eating: A combined neuroimaging and experience sampling study in dieting female college students. Appetite, v. 103, p. 192-199, ago. 2016.

MAGEAU, G. A; BUREAU, J. S.; RANGER, F.; ALLEN, M.; SOENENS, B. The role of parental achievement goals in predicting autonomy-supportive and controlling parenting. Journal of Child Family Studies, v. 25, n. 5, p. 1702-1711, mai. 2016.

MARKLAND, D., RYAN, R. M., TOBIN, V. J., \& ROLLNICK, S. Motivational interviewing and self-determination theory. Journal of Social and Clinical Psychology, v. 24, n. 6, p. 811-831, 2005.

MEURER, S. T.; BENEDETTI, T. R. B.; MAZO, G. Z. Fatores motivacionais de idosos praticantes de exercícios físicos: um estudo baseado na teoria da autodeterminação. Estudos de Psicologia, Natal, v. 17, n. 2. p. 299-304, mai./ago. 2012.

MOLETTA, A. F.; TEIXEIRA, F. A.; FOLLE, A., DO NASCIMENTO, J. V.; DE OLIVEIRA FARIAS, G.; MARINHO, A. Momentos marcantes do estágio curricular supervisionado na formação de professores de educação física. Pensar a Prática, v.16, n.3, set./dez. 2013.

MOREIRA. C. R.; NASCIMENTO JÚNIOR, J. R. A.; MIZOGUCHI, M. V.; VIEIRA, D. V.; VIEIRA, L. F. Impact of adhesion reasons in the motivational regulation of master swimmers during the season. Revista Brasileira de Cineantropometria e Desempenho Humano, v. 18, n. 4, p. 439-440, 2016.

NASCIMENTO, J. V. Escala de auto-percepção de competência profissional em educação física e desportos. Revista Paulista de Educação Física, São Paulo, v. 13, n. 1, p. 5-21, jan./jun. 1999.

NTOUMANIS, N. A self-determination theory perspective on motivation in sport and physical education: Current trends and possible future research directions. In: ROBERTS, G.C.; TREASURE D. C. (Ed.) Motivation in sport and exercise. 3. ed. Champaign, IL: Human Kinetics, 2012, p. 91-128.

OLAFSEN, A. H.; HALVARI, H.; FOREST, J.; DECI, E. L. Show them the money? The role of pay, managerial need support, and justice in a self-determination theory model of intrinsic work motivation. Scandinavian Journal of Psychology, v. 56, n. 4, p. 447-457, ago. 2015. 
PIZANI, J; RINALDI, I. P. B.; MIRANDA, A. C. M.; VIEIRA, L. F. (Des) motivação na educação física escolar: uma análise a partir da teoria da autodeterminação. Revista Brasileira de Ciências do Esporte, v. 38, n. 3, p. 259-266, jul./set. 2016.

RIBEIRO, V. T; FOLLE, A; FARIAS, G. O.; NAZARIO, P. Preocupações pedagógicas e competência profissional de estudantes de educação física em situação de estágio. Revista Educação Física/UEM, Maringá, v. 26, n. 1, p. 59-68, jan./mar. 2015.

RYAN, R. M.; DECI, E. L. Self-determination theory and the facilitation of intrinsic motivation, social development, and well-being. American Psychologist, v. 55, n. 1, p. 68-78, jan. 2000.

RYAN, R. M.; PATRICK, H.; DECI, E. L.; WILLIAMS, G. C. Facilitating health behaviour change and its maintenance: Interventions based on self-determination theory. European Health Psychologist, v. 10, n. 1, p. 2-5, 2008.

SANTOS, K. T.; FERREIRA, L.; BATISTA, R. J.; BITENCOURT, C. T. F.; ARAÚJO, R. P.; CARVALHO, R. B. Percepção discente sobre a influência de estágio extramuro na formação acadêmica odontológica. Revista de Odontologia da UNESP, v. 42, n. 6, p. 420-425, nov./dez. 2013.

SEBIRE, S. J.; JAGO, R.; FOX, K. R.; EDWARDS, M. J.; THOMPSON; J. L. Testing a selfdetermination theory model of children's physical activity motivation: a cross-sectional study. International Journal of Behavioral Nutrition and Physical Activity, v. 10, n. 111, set. 2013.

SILVA JUNIOR, A. P.; FlORES, P. P.; BISCONSINI, C. R.; ANVERSA, A. L. B.; OLIVEIRA, A. A. B. Estágio curricular supervisionado na formação de professores em educação física: uma análise da legislação a partir da resolução CFE 03/1987. Pensar a Prática, v. 19, n. 1, jan./mar. 2016.

STANDAGE, M.; GILLISON, F. B.; NTOUMANIS, N; TREASURE, D. C. Predicting students physical activity and health-related well-being: a prospective cross-domain investigation of motivation across school physical education and exercise settings. Journal of Sport \& Exercise Psychology, v. 34, n. 1, p. 37-46, fev. 2012.

TAYLOR, I. The five self-determination mini-theories applied to sport. In: MELLALIEU S.; HANTON S. (Ed.) Contemporary advances in sport psychology: a review. England: Routledge, 2015. p. 68-90.

VALLERAND, R. J. et al. Construction et validation de l'échelle de motivation en education (EME). Canadian Journal of Behavior Science, v. 21, n. 3, p. 323-49, 1989.

VANSTEENKISTE, M.; RYAN, R. M. On psychological growth and vulnerability: basic psychological need satisfaction and need frustration as a unifying principle. Journal of Psychotherapy Integration, v. 23, n. 3, p. 263-280, set. 2013. 
VIEIRA, L. F.; VIEIRA, J. L. L.; FERNANDES, R. Competência profissional percebida: um estudo com estudantes de educação física em formação inicial. Revista da Educação Física/UEM, Maringá, v. 17, n. 1, p. 95-105, jan./mar. 2006.

Recebido em: 13/03/2017

Revisado em: 26/05/2017

Aprovado em: 07/06/2017

Endereço para correspondência:

jroberto.jrs01@gmail.com

José Roberto Andrade do Nascimento Júnior

Universidade Federal do Vale do São Francisco

Avenida José de Sá Maniçoba

Centro

56304205 - Petrolina, PE - Brasil 